\title{
Strong interaction of a turbulent spot with a shock-induced separation bubble
}

\author{
L. Krishnan and N. D. Sandham ${ }^{\text {a) }}$ \\ Aerodynamics and Flight Mechanics Research Group, School of Engineering Sciences, \\ University of Southampton, Southamptom SO17 1BJ, United Kingdom
}

(Received 9 June 2006; accepted 4 December 2006; published online 25 January 2007)

\begin{abstract}
Direct numerical simulations have been conducted to study the passage of a turbulent spot through a shock-induced separation bubble. Localized blowing is used to trip the boundary layer well upstream of the shock impingement, leading to mature turbulent spots at impingement, with a length comparable to the length of the separation zone. Interactions are simulated at free stream Mach numbers of two and four, for isothermal (hot) wall boundary conditions. The core of the spot is seen to tunnel through the separation bubble, leading to a transient reattachment of the flow. Recovery times are long due to the influence of the calmed region behind the spot. The propagation speed of the trailing interface of the spot decreases during the interaction and a substantial increase in the lateral spreading of the spot was observed. A conceptual model based on the growth of the lateral shear layer near the wingtips of the spot is used to explain the change in lateral growth rate.

(C) 2007 American Institute of Physics. [DOI: 10.1063/1.2432158]
\end{abstract}

\section{INTRODUCTION}

Understanding and prediction of compressible boundary layer flow physics, including laminar-to-turbulent transition and shock interactions, is of great importance in the design of flight vehicles for supersonic and hypersonic speeds. By itself, transition is difficult to predict and the presence of shock waves brings additional complexity.

Natural transition in a laminar flow often leads to localized regions of turbulent flow known as turbulent spots $\left(\right.$ Emmons $\left.{ }^{1}\right)$. The growth and merging of these spots results in a fully developed turbulent flow field, with the length of the transition region mainly depending on spot characteristics such as lateral growth rate and convective speeds of the leading and trailing edges, as well as on interactions between spots (Dhawan and Narasimha ${ }^{2}$ ). Schubauer and Klebanoff et $a .^{3}{ }^{3}$ estimated convective speeds of the front and the rear interfaces of the spot as 0.88 and 0.50 of the free-stream velocity, respectively. A detailed review of this type of transition process was given by Narasimha, ${ }^{4}$ covering turbulent spots in a variety of flows and a generalized intermittency distribution function based on the hypothesis of concentrated breakdown was developed.

The structure of turbulent spots has been examined in detail in low speed flows (Schubauer and Klebanoff et al., ${ }^{3}$ Wygnanski et al., ${ }^{5}$ Seifert and Wygnanski, ${ }^{7}$ Gutmark and Blackwelder ${ }^{6}$ ). The basic arrowhead spot structure has a leading-edge overhang, a turbulent core, and a "calmed" region behind the spot where fast-moving fluid is directed towards the wall. Laser-Doppler velocity measurements of Cantwell et al. ${ }^{8}$ showed strong entrainment of undisturbed fluid along the outer part of the spot tail and also near the front overhang, while flow visualizations showed longitudinal structures in the sublayer. Spots are generally assumed to

a)Electronic mail: n.sandham@soton.ac.uk grow in the lateral direction due to a destabilization of the surrounding laminar boundary layer (Gad-El-Hak et al. ${ }^{9}$ ).

Narasimha ${ }^{4}$ reported the influence of pressure gradients on spot geometry, propagation characteristics, and spreading rate; an adverse pressure gradient was shown to have a destabilizing effect, while a favorable pressure gradient delays the transition process. This has been confirmed in later work, including Katz et al. ${ }^{10}$ for a favorable pressure gradient boundary layer and Seifert and Wygnanski, ${ }^{7}$ who found a doubling of spot growth rate in a strong adverse pressure gradient flow. Measurements based on surface heat transfer Zhong et al. ${ }^{11}$ also showed a significant increase in spot lateral spreading rates with increasing adverse pressure gradients, although the growth angles measured in this way are smaller than those based on velocity measurements.

Data for compressible spots is much more limited. Early data for the variation of turbulent spot spreading angles with local Mach number was summarized by Fischer. ${ }^{12}$ The spreading angle relative to the wall was seen to remain invariant with Mach number $M$, while the lateral halfspreading angle decreased sharply from $10-11^{\circ}$ to $3^{\circ}$ with increasing Mach number up to $M=6$. Clark et al. ${ }^{13}$ measured the celerities of naturally occurring turbulent spots to study the effects of compressibility and favorable pressure gradients. Their estimated lateral half-spreading angle at $M$ $=1.32$ was $6^{\circ}$, already well below the $9.9^{\circ}$ measured in the same rig at $M=0.24$. More recent boundary layer transition measurements by $\mathrm{Mee}^{14}$ in hypervelocity flows (Mach 6) also showed that turbulent spots grow with a much lower lateral spreading angle than at low Mach numbers.

In aerodynamic configurations like ramps (compression corners) and supersonic intakes, significant shock/boundarylayer interactions can occur. If the interacting boundary layer is transitional, this may result in a highly unsteady and complex flow field. Forced instability waves have been considered in the work of Pagella et al. ${ }^{15}$ Here we consider direct 
numerical simulation (DNS) of the dynamics of a mature turbulent spot interacting with a separation bubble arising from impingement of an oblique shock wave. The mutual interaction effects are strong, including bubble collapse and a sharp increase in spot lateral spreading rate. The results are used to discuss a simplified mechanism of lateral spot growth rate, which parameterizes the spot growth rate with properties of the lateral laminar-turbulent interface.

\section{NUMERICAL APPROACH}

\section{A. Governing equations}

The nondimensional Navier-Stokes (N-S) equations that govern the unsteady, compressible flows in Cartesian coordinates are

$$
\begin{aligned}
& \frac{\partial \rho}{\partial t}+\frac{\partial \rho u_{j}}{\partial x_{j}}=0, \\
& \frac{\partial \rho u_{i}}{\partial t}+\frac{\partial \rho u_{i} u_{j}}{\partial x_{j}}=-\frac{\partial p}{\partial x_{i}}+\frac{1}{\operatorname{Re}} \frac{\partial \tau_{i j}}{\partial x_{j}}, \\
& \frac{\partial E}{\partial t}+\frac{\partial(E+p) u_{j}}{\partial x_{j}}=\frac{\partial u_{i} \tau_{i j}}{\partial x_{j}}+\frac{1}{(\gamma-1) \operatorname{Re} \operatorname{Pr} M^{2}} \frac{\partial}{\partial x_{j}}\left(\mu \frac{\partial T}{\partial x_{j}}\right),
\end{aligned}
$$

where

$$
\tau_{i j}=\mu\left(\frac{\partial u_{j}}{\partial x_{i}}+\frac{\partial u_{i}}{\partial x_{j}}-\frac{2}{3} \delta_{i j} \frac{\partial u_{k}}{\partial x_{k}}\right) .
$$

The temperature is given by

$$
T=\gamma(\gamma-1) M^{2}\left(\frac{E}{\rho}-\frac{1}{2} u_{i} u_{i}\right) .
$$

The equation of state can be written as

$$
p=(\gamma-1)\left(E-\frac{1}{2} \rho u_{i} u_{i}\right)=\frac{\rho T}{\gamma M^{2}} .
$$

The nondimensional parameters governing the flow are Reynolds number $\operatorname{Re}=\rho_{r}^{*} u_{r}^{*} \delta_{r}^{*} / \mu_{r}^{*}$; the Mach number $M=u_{r}^{*} / \sqrt{\gamma R^{*} T_{r}^{*}}$; the ratio of specific heats $\gamma=c_{p}^{*} / c_{v}^{*}$; and the Prandtl number $\operatorname{Pr}=c_{p}^{*} \mu^{*} / k^{*}$, which is set to 0.72 . The variation of the dynamic viscosity with temperature is accounted for by using a power law $\left(\mu^{*} / \mu_{r}^{*}=\left(T^{*} / T_{r}^{*}\right)^{\omega}\right)$ with a constant $\omega$ value of 2/3. In the above expressions the subscript $r$ denotes a reference value and asterisks $\left(^{*}\right)$ represent dimensional variables.

\section{B. Entropy-splitting}

The governing equations are solved using a stable highorder scheme in which an entropy splitting is applied to the Euler terms. The method is formulated as a system of hyperbolic conservation equations

$$
u_{t}+f_{x}=0 \text {, }
$$

where $u$ and $f$ are column vectors. Applying the entropy variable transformation using the split high-order entropy conserving scheme of Gerritsen and Olsson ${ }^{16}$ gives

$$
u_{w} w_{t}+f_{w} w_{x}=0 .
$$

The final split form can be written as

$$
\frac{\beta}{\beta+1} u_{t}+\frac{1}{\beta+1} u_{w} w_{t}+\frac{\beta}{\beta+1} f_{x}+\frac{1}{\beta+1} f_{w} w_{x}=0,
$$

where $\beta(\neq-1)$ is a splitting parameter, chosen as $\beta=4.0$ here. The original unsplit conservative form can be recovered as $\beta \rightarrow \infty$.

The spatial discretization is carried out using a fourthorder central-difference scheme while the time integration uses a third-order Runge-Kutta method. A stable boundary scheme of Carpenter et al. ${ }^{17}$ along with a Laplacian formulation of the viscous and heat conduction terms from Sandham et al., ${ }^{19}$ are used to prevent any odd-even decoupling associated with central schemes. An artificial compression method (ACM) variant of a standard total variation diminishing (TVD) family is used to capture flow discontinuities. The TVD filter is applied at the end of each full time step in the form of an additional numerical flux term

$$
F_{j+(1 / 2)}=R_{j+(1 / 2)} \Phi_{j+(1 / 2)} \Psi_{j+(1 / 2)},
$$

where $R$ is the right eigenvector matrix of the flux Jacobian from the Euler equations and $\Phi$ is defined by the TVD scheme. $\Psi$ is a sensor (Ducros et al. ${ }^{18}$ ) which takes low values where the flow is turbulent and values close to one in the vicinity of a shock,

$$
\Psi=\frac{(\nabla \cdot \mathbf{V})^{2}}{(\nabla \cdot \mathbf{V})^{2}+\omega^{2}+\epsilon},
$$

where $\boldsymbol{V}$ is the velocity vector, $\omega$ is the vorticity amplitude, and $\epsilon$ is machine zero. More details regarding the entropysplitting and other numerical issues used in the present scheme can be found in Sandham et al. ${ }^{19}$ The present numerical implementation has been validated for various test cases including shock impingement and fully turbulent flow by Krishnan. ${ }^{20}$

\section{SIMULATION DETAILS}

\section{A. Computational domain and boundary conditions}

The configuration for the simulations is shown in Fig. 1. All the lengths are normalized with the displacement thickness $\left(\delta_{r}^{*}=\delta_{\text {in }}^{*}\right)$ of the laminar inflow profile. The laminar base flow is obtained by a separate self-similar compressible boundary layer solution. In the supersonic part of the inflow all the properties are fixed, while in the subsonic region the pressure is extrapolated from the interior.

Boundary conditions consist of characteristic-based nonreflective conditions at the outlet, an integrated characteristic boundary condition at the top surface and a no-slip, isothermal condition at the flat plate surface $\left(\operatorname{Krishnan}^{20}\right)$. Periodic boundary conditions are applied in the spanwise direction.

The flow domain is discretized using an equally spaced grid along the streamwise $(x)$ and spanwise $(z)$ directions and a stretched grid in the wall normal $(y)$ direction. The mapping function used for the grid stretching is 


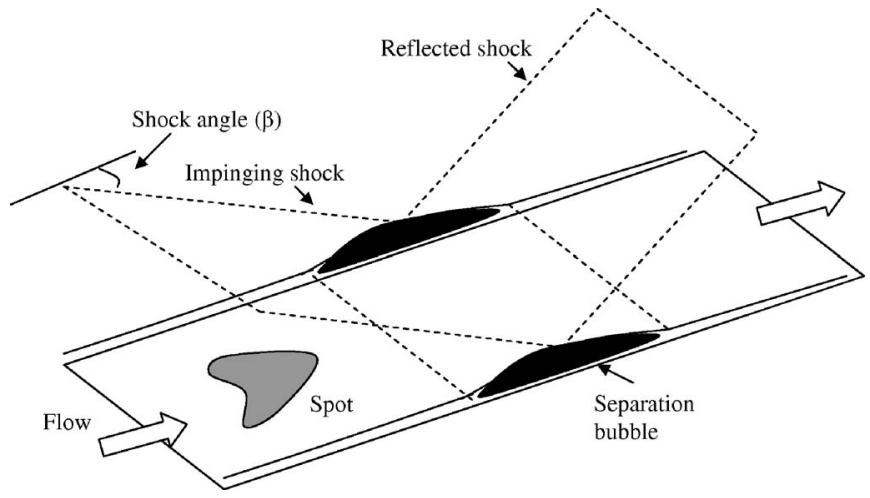

FIG. 1. Cartoon illustrating the spot/oblique shock-induced separation bubble interaction.

$$
y_{j}=\frac{\sinh \left(b_{y} \eta_{j}\right)}{\sinh \left(b_{y}\right)},
$$

where

$$
\eta_{j}=L_{y} \frac{(j-1)}{\left(N_{y}-1\right)} ; \quad j=1, N_{y}
$$

$N_{y}$ is the number of grid points in the wall-normal direction and $L_{y}$ is the height of the domain. The stretching parameter in the present simulations is set to $b_{y}=5.0$.

\section{B. Simulation parameters}

Details of the simulation parameters are given in Table I. The simulations M2S and M4S refer to turbulent spot simulations without shock interaction, while M2SS and M4SS refer to shock/spot interaction simulations. For the given flow conditions it was verified that an unperturbed base flow with a shock-induced separation bubble remains laminar. In the M2SS case an oblique shock was introduced on the upper boundary with an incident shock angle of $32.58^{\circ}$ such that it impinges approximately at $x=137$ at the lower boundary in the absence of a boundary layer. For this angle, the static pressure ratio, i.e., the ratio of pressure after the shock reflection to pressure before the shock impingement, is $p_{3} / p_{1}$ $=1.4$. In the M4SS case an oblique shock with an incident shock angle of $17^{\circ}$ impinging at a streamwise location $x=206$ with a static pressure ratio of $p_{3} / p_{1}=1.99$ is considered. The reduction in the free-stream velocity $\left(u_{\infty}\right)$ after the shock reflection is about $3 \%$ and $2 \%$ for the M2SS and M4SS cases, respectively. First the laminar base flow is allowed to develop along the plate until steady-state conditions are reached. Then the shock conditions are imposed at the upper boundary. The solution is advanced until a steady laminar separation bubble was obtained. The calculations

TABLE I. Simulation details.

\begin{tabular}{lccccc}
\hline \hline Case & Mach & $\operatorname{Re}_{\delta_{\text {in }}^{*}}$ & $T_{w} / T_{\infty}$ & $L_{x}, L_{y}, L_{z}$ & $N_{x}, N_{y}, N_{z}$ \\
\hline M2S & 2 & 950 & 1.672 & $400 \times 60 \times 60$ & $801 \times 101 \times 121$ \\
M2SS & 2 & 950 & 1.672 & $250 \times 60 \times 60$ & $501 \times 101 \times 121$ \\
M4S and M4SS & 4 & 2000 & 3.694 & $450 \times 60 \times 60$ & $801 \times 101 \times 121$ \\
\hline \hline
\end{tabular}

were performed up to nondimensional times of 7000 and 10000 for the Mach 2 and Mach 4 cases, respectively.

Grid refinement study for the laminar base flows showed convergence even with fewer grid points (Figs. 3 and 4). For the grids shown in Table I, the streamwise and spanwise resolutions in viscous wall units in the spot cases are found to be $\Delta x^{+}, \Delta z^{+}=7.5-12.5$ based on the maximum local mean friction velocity $\left[u_{\tau}=\sqrt{\mu_{w}(d u / d y)_{w} / \rho_{w}}\right.$, with subscript $w$ denoting the wall]. Mean flow properties in the spot core are found by averaging over $20 \leq z \leq 40$. Typically 11 points are used in the sublayer region $\left(y^{+}<10\right)$ and 27 points within $y^{+}=30$. These values can be compared with Spalart $^{21}$ who performed a DNS of a spatially evolving turbulent boundary layer with grid resolutions $\Delta x^{+}=20, \Delta z^{+}=7.0$. The spanwise resolution in the $\mathrm{M} 2 \mathrm{~S}$ case seems to be slightly coarse in comparison to the spanwise resolution of Spalart. ${ }^{21}$ This is also evident from inspection of higher derivatives of flow variables. In order to assess the influence of the spanwise grid resolution on the spot growth, a check was performed by doubling the number of grid points in the spanwise direction for the M2S case. This fine grid simulation was carried out up to time $t=249$ in a computational box of size $400 \times 60$ $\times 50(x, y, z)$ with $801 \times 101 \times 201\left(N_{x}, N_{y}, N_{z}\right)$ grid points. The results showed no variation in the locations of the spot's front, tail and lateral boundaries and the shape $\left(\operatorname{Krishnan}^{20}\right)$. This suggests that the spanwise grid resolution employed for the M2S case is adequate for these properties.

\section{Spot trigger}

The spots are triggered upstream of the bubble using a localized blowing trip. The laminar base flow is perturbed by a localized injection of low momentum fluid through the plate surface. A spanwise symmetric rectangular slot of dimensions $4 \times 4 \quad(20 \leq x \leq 24,28 \leq z \leq 32)$ was used. The blowing trip was applied for a short duration of 8 nondimensional time units $\left(\delta_{\text {in }}^{*} / u_{\infty}\right)$ by specifying vertical velocity at the plate surface as

$$
v_{\text {inj }}=A u_{\infty} .
$$

A large amplitude $A=0.2$ of the disturbance is chosen $\left(\right.$ Elder $^{22}$ ) such that a spot can be triggered and studied within the present domain size.

\section{RESULTS}

\section{A. Base flow without turbulent spot}

The impinging oblique shock introduces a pressure jump near the impingement location. If the pressure increase across the shock wave is sufficiently strong then the laminar boundary layer separates. The impinging oblique shock is reflected initially as a system of expansion fans and shock waves, which deflect the flow towards the wall and cause boundary-layer reattachment and hence a closed separation bubble is formed. Isodensity contours given in Fig. 2 show the flow structure for both Mach 2 and Mach 4 cases. Note that the vertical scale in Fig. 2 has been expanded to show the bubble clearly; in reality the bubbles are very shallow. The shock strengths and the impingement locations for the 


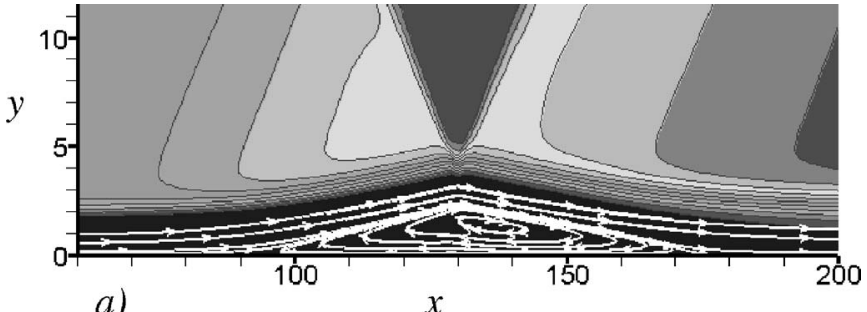

a)

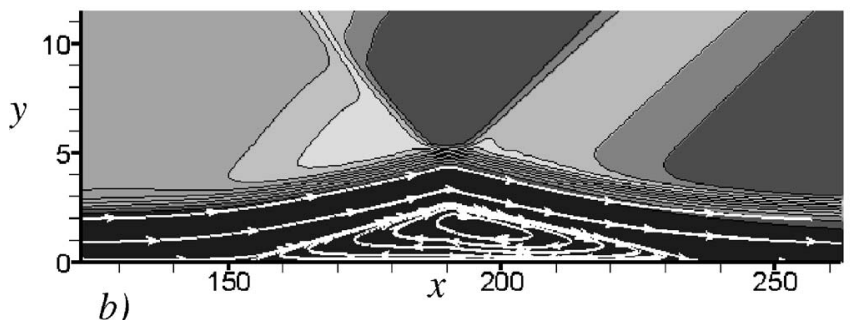

FIG. 2. Isodensity contours superimposed with streamline traces showing the separation bubble, (a) Mach 2 bubble, (b) Mach 4 bubble.

two cases are selected so as to get a bubble length comparable to the length of the turbulent spot at the beginning of the interaction. The surface skin friction distributions, shown in Figs. 3 and 4 for the M2SS and M4SS cases, respectively, show the extent of the separation zone. The minimum skin friction is observed towards the rear of the bubble. Wall static pressure distributions are given in Figs. 5 and 6 for the M2SS and M4SS cases, respectively. The absence of a well defined pressure plateau suggests a moderate viscousinviscid interaction for the present shock strengths and Mach numbers. Separation bubble parameters are given in Table II. Length-to-height aspect ratios $l_{b} / h_{b}$ of the separation bubbles are of the order of 30 .

\section{B. Structure of a spot}

In the absence of shock interaction the localized disturbance evolves into a classical spot structure, as illustrated by Fig. 7 at time $t=249$ which shows the isosurfaces of the second invariant of the velocity gradient tensor

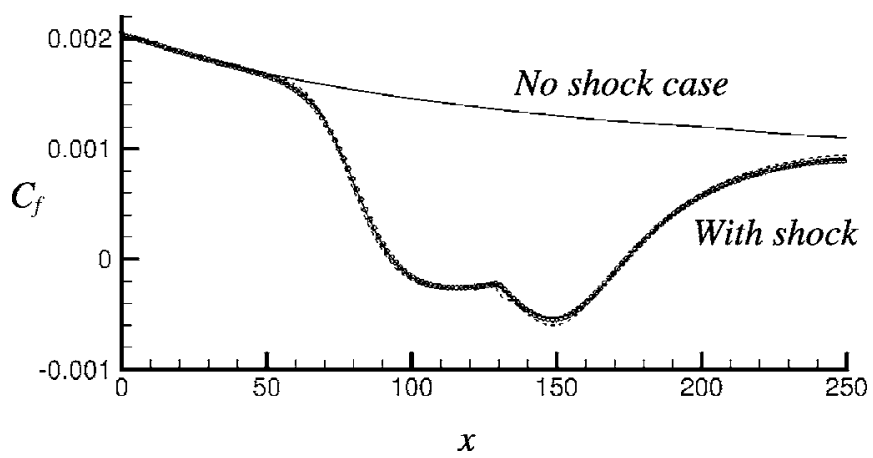

FIG. 3. Skin friction distribution along the flat plate surface (M2SS). Solid line, $501 \times 101$; symbol, $161 \times 121$; dashed line, $81 \times 81$.

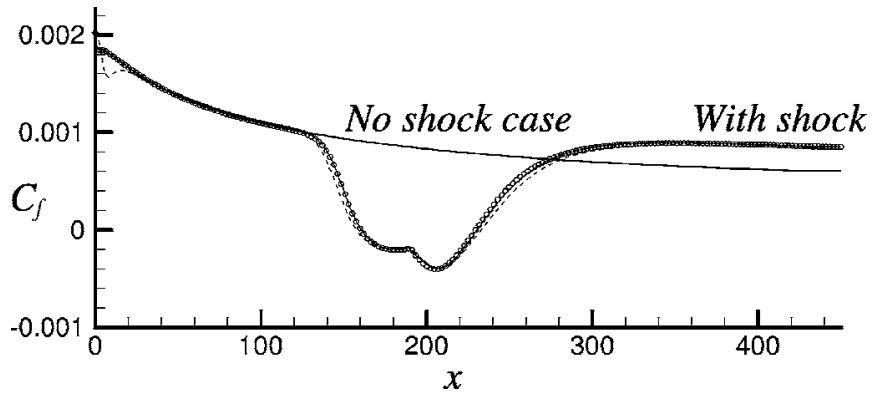

FIG. 4. Skin friction distribution along the flat plate surface (M4SS). Solid line, $801 \times 101$; symbol, $321 \times 121$; dashed line, $161 \times 81$.

$$
\Pi=\frac{\partial u_{i}}{\partial x_{j}} \frac{\partial u_{j}}{\partial x_{i}} .
$$

This picture shows an arrowhead shape, with a leading-edge overhang and a turbulent core. Hairpin structures can be seen near the leading edge of the spot, reminiscent of the conceptual picture of Perry et al. ${ }^{23}$ Quasistreamwise vortices are evident at the lateral extremities of the spot (see Sec. V for an explanation of their origin).

Contour plots of the streamwise velocity and temperature at $M=2$ (integrated across the spot width) relative to the laminar undisturbed value are shown in Figs. 8(a) and 8(b), respectively, to identify the overall structure of the spots. The excess streamwise velocity region and temperature deficit near the rear of the spot confirms the presence of a sweeping process involving an inrush of cold fluid towards the wall, as in Wygnanski et al. ${ }^{26}$ Positive temperature perturbations correspond to fluid from the near wall region, while negative perturbations indicate fluid from the outer region. Maximum surface heat transfer thus occurs near the rear of the spot. A velocity deficit and temperature excess near the front of the spot shows the ejection of hot near wall fluid resulting in the formation of the front overhang. Entrainment of cold outer fluid leads to the tongue-like protrusion of the temperature deficit region below the front overhang. A similar structure was found at $M=4$.

\section{Spot "tunnelling"}

The effect of spot interaction with the shock-induced separation bubble can be seen in Figs. 9 and 10. In each case a plan view of the second invariant $(\Pi=-0.0008)$ compares the spot structure without interaction (the upper figure in each case) with the spot structure undergoing interaction

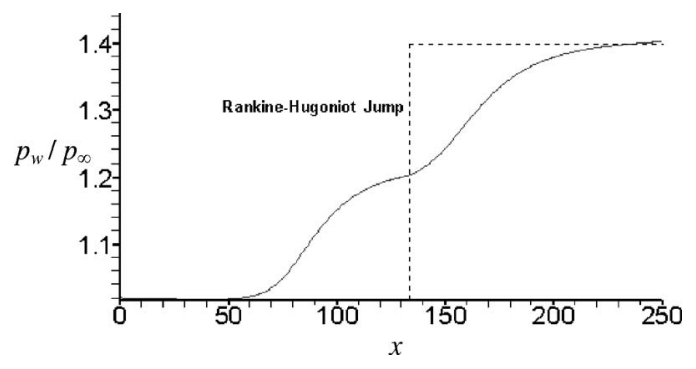

FIG. 5. Static pressure distribution along the flat plate surface (M2SS). 


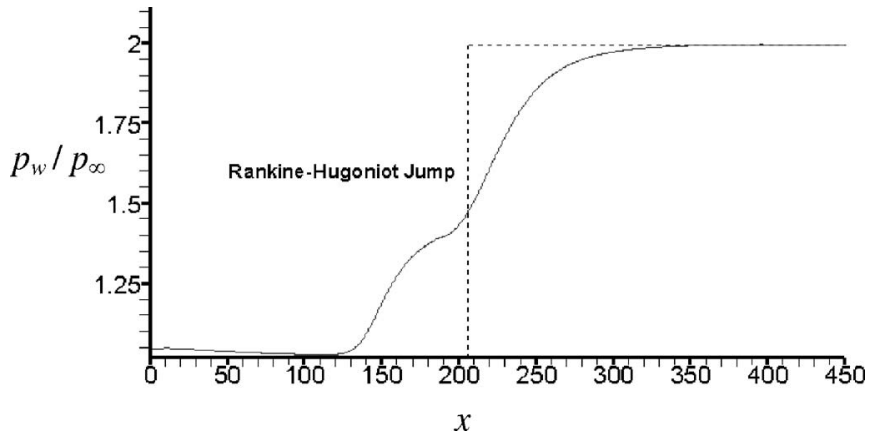

FIG. 6. Static pressure distribution along the flat plate surface (M4SS).

(lower figure). At Mach 2 the center of the spot is close to the reattachment location, while at Mach 4 the wingtip of the spot is at reattachment. It can be seen that the location of the leading edge of the spot is not strongly affected by the interaction, while the trailing edge is retarded, leading to an increase in spot length. There is proportionately a much larger lateral spreading of the spot, leading to spots with reduced length-to-width aspect ratios. Interaction of the oblique shock and the recirculation zone with the spot substructures can be seen in Figs. 11 and 12, where the spanwise-coherent structure is the core of the recirculation region. In comparison to the isolated spot structure shown in Fig. 7, the spot has grown significantly in the spanwise direction, with a multitude of new flow structures in the expanded spot core. This growth suggests a strong destabilization of the surrounding laminar boundary layer along the wingtips of the spot due to the bubble interaction. Large scale streamwise structures continue to be seen along the rear interface of the spot. Figure 13 (M4SS at $t=417$ ) shows the generation of streamwise structures behind the spot, as the spot rear interface passes through the reattachment location.

The collapse of the bubble due to the spot passage can be observed clearly by looking at the zero contour of wall shear $\left[(d u / d y)_{w}=0\right]$ at the flat plate surface, shown in Figs. 14 and 15 for $M=2$ and $M=4$, respectively. For $M=2$ the spot enters the upstream interface of the separation bubble just before $t=139$ and leaves just before $t=413$. Since the front overhang is located away from the wall, at a height that is greater than the height of the separation bubble, its interaction with the bubble is negligible. The core of the spot interacts with the bubble at $t=194$. Shortly afterwards the core of the spot collapses the bubble by "tunnelling" through the separation region. At $t=255$ a complete collapse of the bubble around the mid span region is seen with almost no regions of reversed flow. The re-establishment of the bubble and the upstream interface of the calmed region inside the bubble can be noticed at $t=413$. The Mach 4 spot/bubble interaction shows a similar effect due to spot passage

TABLE II. Details of the bubble.

\begin{tabular}{lccccc}
\hline \hline Case & $l_{b}$ & $h_{b}$ & $l_{b} / h_{b}$ & $\delta_{\text {separation }}^{*}$ & $\delta_{\text {reattachment }}^{*}$ \\
\hline M2SS & 80.5 & 2.35 & 34.30 & 1.796 & 1.867 \\
M4SS & 81.0 & 2.60 & 31.20 & 2.426 & 2.209 \\
\hline \hline
\end{tabular}

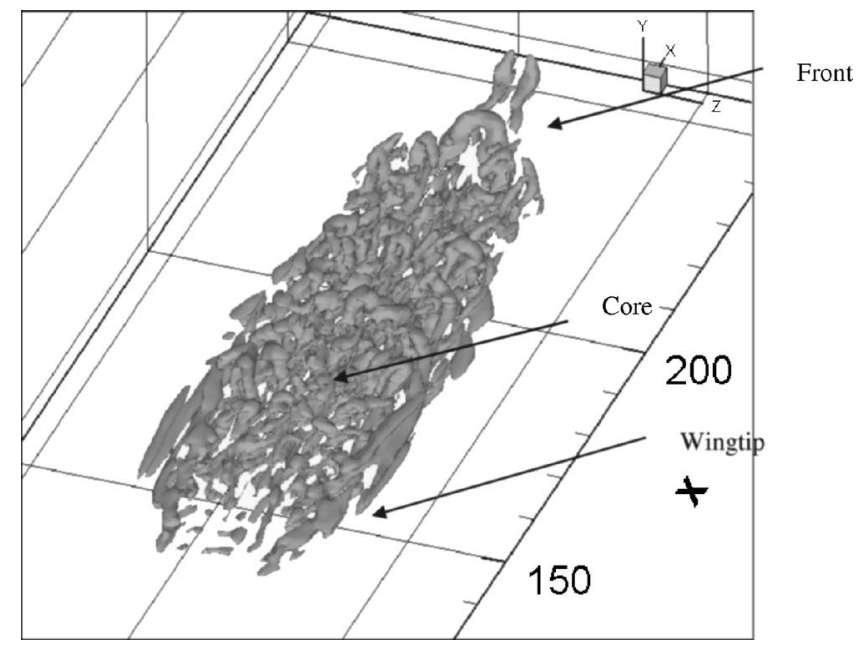

FIG. 7. Isosurface of the second invariant of the velocity gradient tensor at $t=249$; Mach 2 isolated spot without shock impingement $(\Pi=-0.0008)$.

through the bubble (Fig. 15). Since the disturbances are contained within the spot and are convecting downstream at the spot propagation speed, the bubble slowly re-establishes after the spot passage, as in the Mach 2 interaction case. This bubble re-establishment can be clearly seen in the wall shear contour at $t=417$.

Figure 16 quantifies the variation of the wall shear within the bubble at various time instants during the spot/ bubble interactions. At any location within the bubble during the spot passage, the spot front portion arrives first, then the turbulent spot core and finally the spot "calmed" region, where disturbances reduce to zero. In the figure, zone A locates the time range during which the overhang region traverses the bubble, but the wall shear stress is relatively unaffected. The following zone B exhibits a strong change in wall shear and extends until the turbulent core of the spot passes the laminar reattachment line. Zone $\mathrm{C}$ covers the slow relaxation of the separation bubble, which is still under the influence of the calmed region behind the spot. In this region there is a strong inflow of high momentum fluid, which serves to damp any upstream diffusion of turbulence and
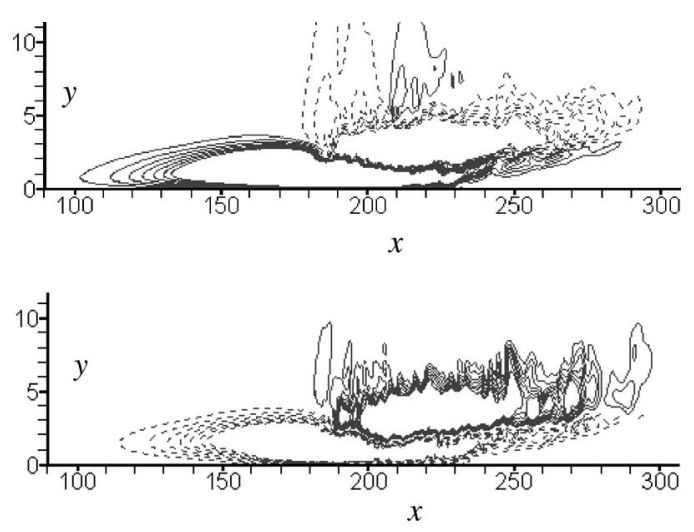

FIG. 8. Integrated perturbations contours at $t=300(M=2)$; dashed contours: negative values, solid: positive values. (a) $u^{\prime}=\int\left(u-u_{l}\right) d z$, (b) $T^{\prime}=\int\left(T-T_{l}\right) d z$. The subscript $l$ refers to the surrounding laminar flow and the integrals are carried out over local spot width. 

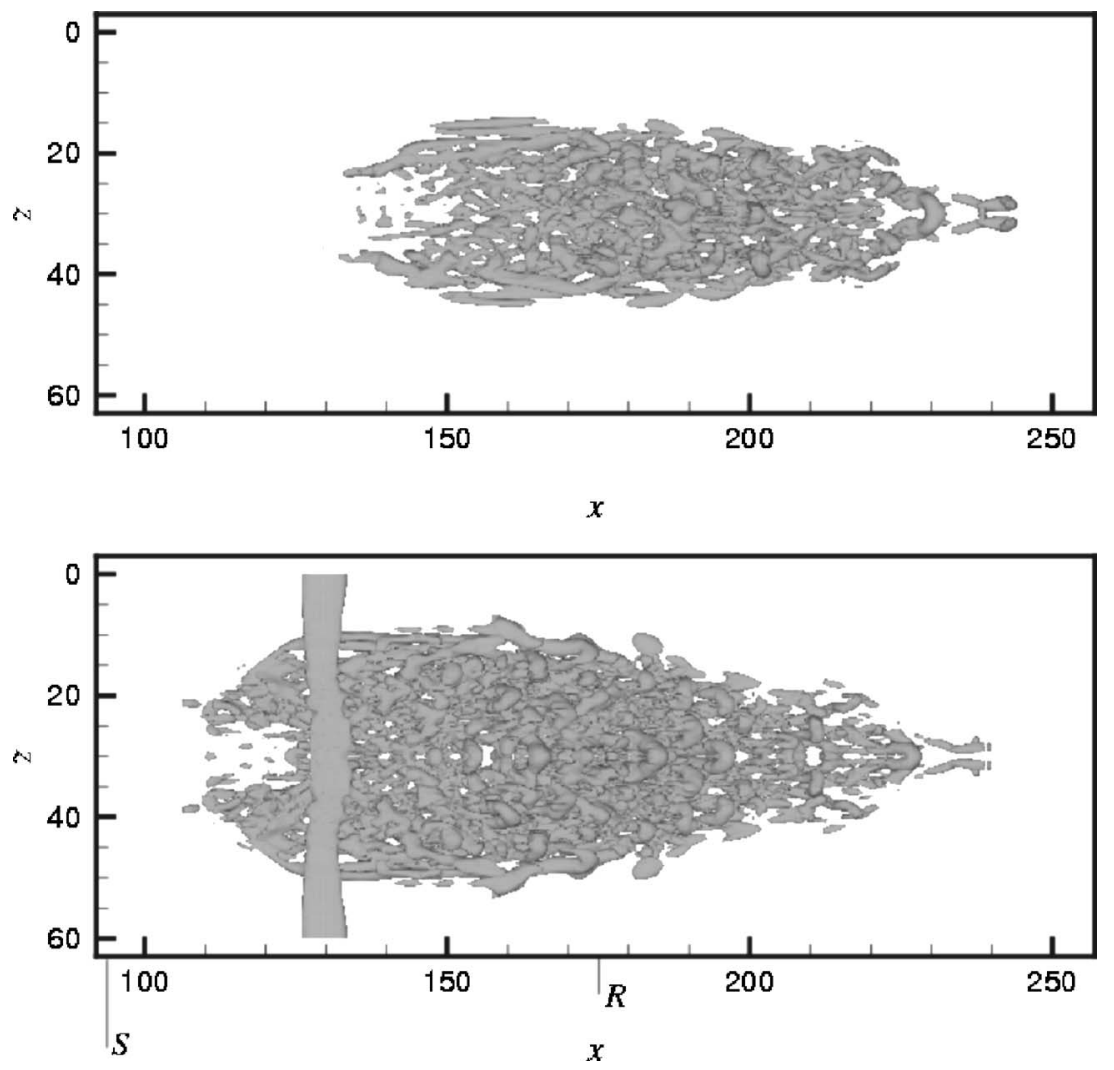

FIG. 9. Isosurface of the second invariant of the velocity gradient tensor $(\Pi=-0.0008)$ comparing the spot growth without and with shock interaction at Mach 2. Top figure, no shock, $t=249$; bottom figure, with shock interaction, $t=255, \mathrm{~S}$ is the separation location, and $\mathrm{R}$ is the reattachment location.

prevent new instabilities in the laminar boundary layer behind the spot. The effect of this sweeping motion (positive streamwise velocity fluctuation and negative wall-normal velocity fluctuation) is to increase the wall shear stress and delay the reappearance of separated flow. Such a collapse and slow recovery of separated regions by turbulent patches is exploited in turbomachinery applications. For example Hodson and Howell ${ }^{24}$ noticed that periodic wakes passing a separation bubble reduced the profile loss. The reason for this reduction in the drag can be attributed to the slow recovery from the bubble collapse.

\section{Spot celerities and growth rates}

Three-dimensional isosurfaces of wall-normal vorticity with a threshold value of \pm 0.06 are used to identify the spot envelope. The propagation speeds of the front and the rear interfaces of the spot and the lateral spreading rates are obtained by tracking the boundaries of the spots at various time instants during their evolution. Estimated spot celerities are presented in Table III. The lateral half-spreading angle obtained for the M2S and the M4S cases without the bubble interaction are in agreement with the data of Fischer. ${ }^{12}$ The
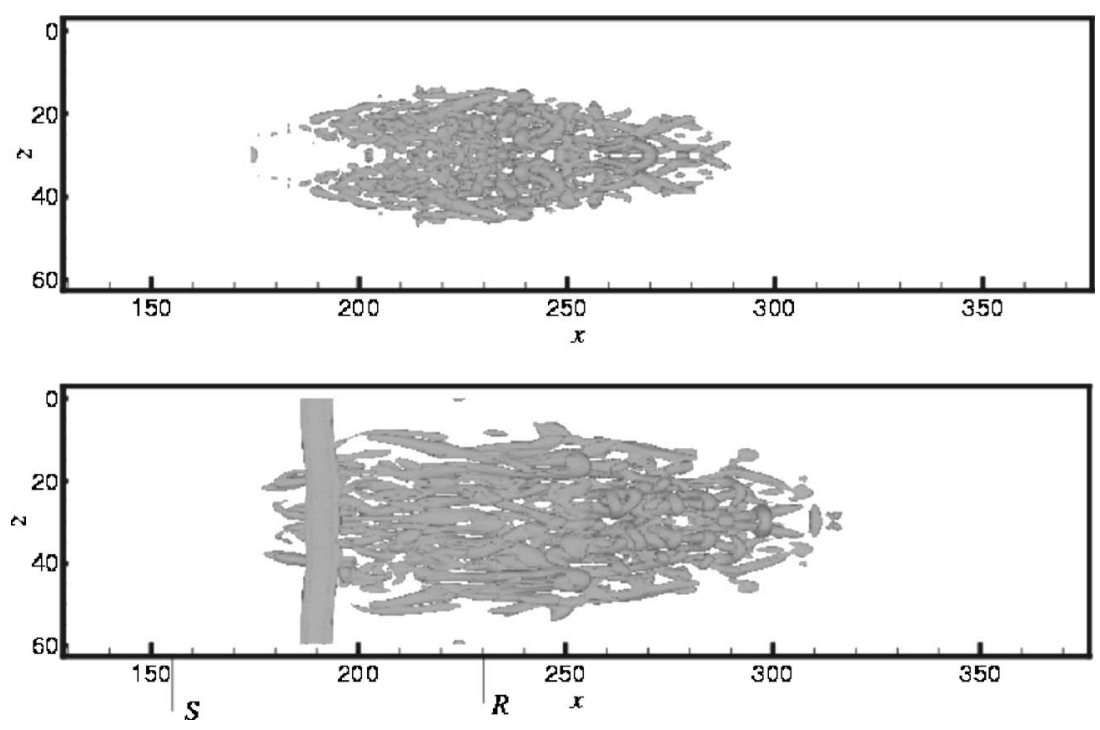

FIG. 10. Isosurface of the second invariant of the velocity gradient tensor ( $\Pi=-0.0008)$ comparing the spot growth without and with shock interaction at Mach 4. Top figure, no shock, $t=310$; bottom figure, with shock interaction, $t=330, \mathrm{~S}$ is the separation location, and $\mathrm{R}$ is the reattachment location. 


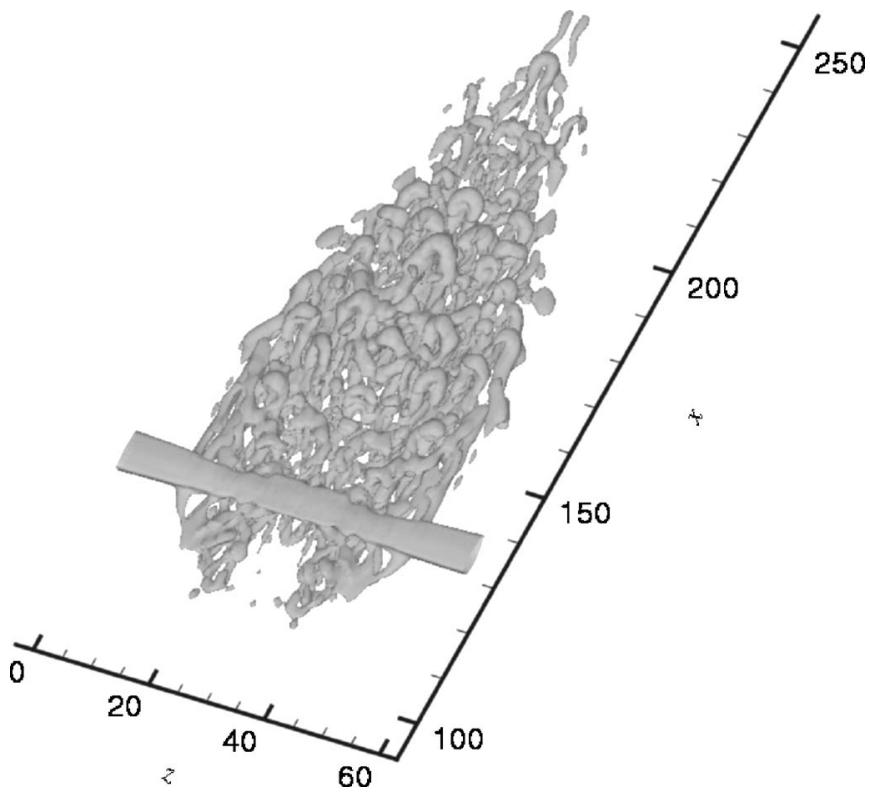

FIG. 11. Spot substructures at $t=255$; Mach 2 spot/bubble interaction $(\Pi=-0.0008)$.

front convection speeds of spots in the M2SS and M4SS cases are 0.86 in both cases, compared to a front convection speed of 0.87 in both the isolated spot cases. This confirms the fact that the front overhang, which is away from the wall, plays no significant role in the bubble interaction process. The calculated rear convection speeds for the M2SS and M4SS cases are 0.42 and 0.45 , respectively. This reduction in the tail convective speed of the spots in comparison with the isolated spots ( 0.53 and 0.59 , respectively) is an indication of the interaction with the slow-moving fluid near the surface under the separation bubble.

While tunnelling through the bubble the spots show enhanced growth in the lateral direction, shown in Figs. 17 and

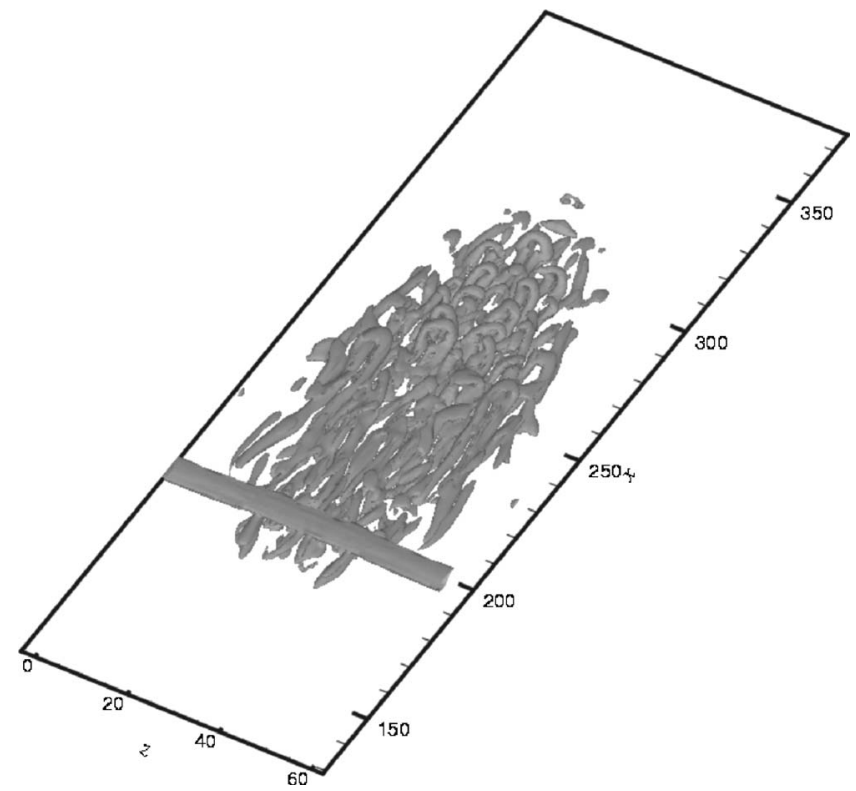

FIG. 12. Spot substructures at $t=330$; Mach 4 spot/bubble interaction ( $\Pi$ $=-0.0008)$.

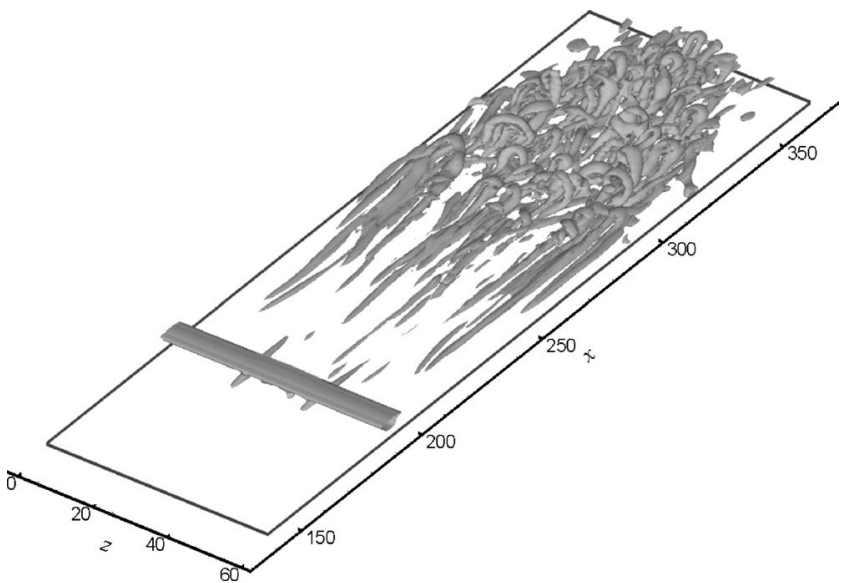

FIG. 13. Spot substructures at $t=417$ showing the streamwise structures near the rear interface; Mach 4 spot/bubble interaction $(\Pi=-0.0008)$.

18 for $M=2$ and $M=4$, respectively. The spot growth is not self-similar in the interaction region. The lines shown in Figs. 17 and 18 and the numbers in Table III indicate the spot growth trends in the interaction zone. The lateral half-

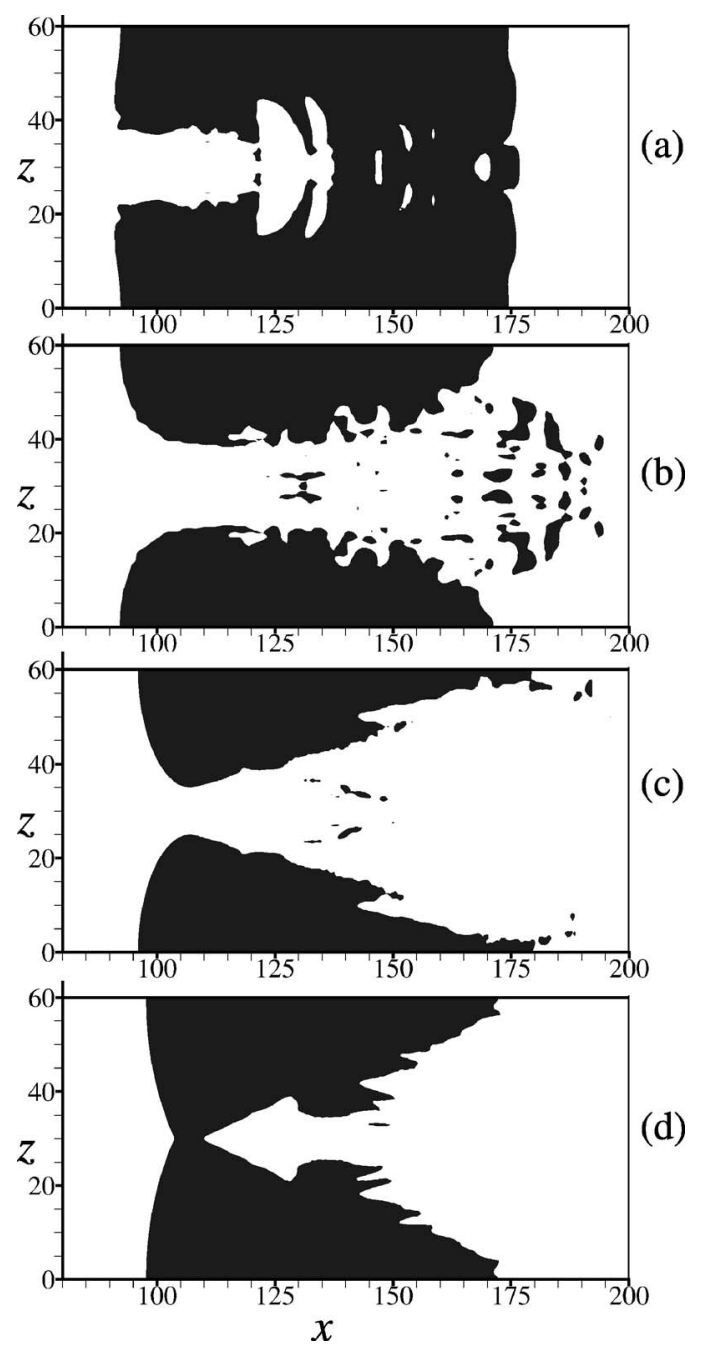

FIG. 14. Zero contour of the wall shear; $(d u / d y)_{w}=0$ demonstrating the tunnelling of the bubble due to the spot passage (M2SS). (a) $t=194$, (b) $t=255$, (c) $t=350$, (d) $t=413$. 


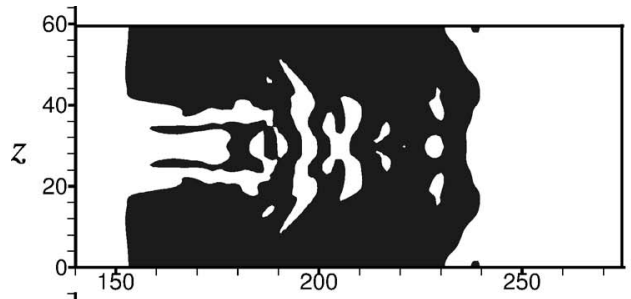

(a)

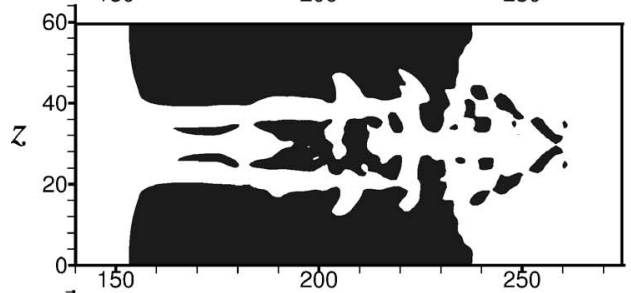

(b)

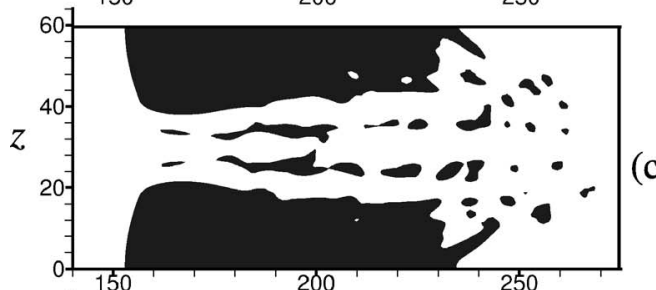

(c)

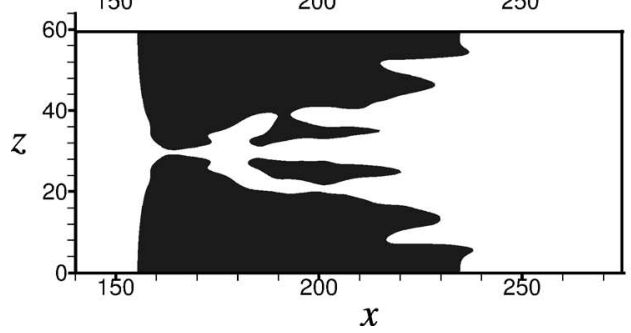

(d)

FIG. 15. Zero contour of the wall shear; $(d u / d y)_{w}=0$ demonstrating the tunnelling of the bubble due to the spot passage (M4SS). (a) $t=256$, (b) $t=300$, (c) $t=330$, (d) $t=417$.

spreading angle for the M2SS case is about $18.5^{\circ}$ while for the M4SS case it is around $11^{\circ}$, both of which are significantly higher (by a factor of 3-4) than without the interaction (see Table III).

The observed variations in the spot celerities due to the spot/bubble interactions are consistent with the experimental results in a low-speed adverse pressure gradient flow reported by Seifert and Wygnanski. ${ }^{7}$ They observed the lateral half-spreading angle to increase from $10^{\circ}$ to $21^{\circ}$ for a strong adverse pressure gradient relative to zero pressure gradient

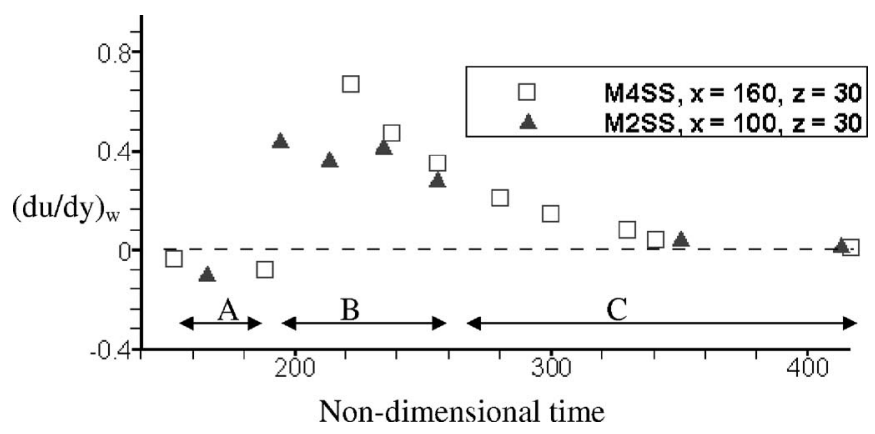

FIG. 16. Temporal evolution of wall-shear $(d u / d y)_{w}$ within the bubble during the spot passage. (A) Interaction of spot front; (B) interaction of spot core; (C) interaction of the calmed region.
TABLE III. Front and rear convection speeds and lateral spreading rate of spot-alone (M2S and M4S) and spot/shock interaction (M2SS and M4SS) simulations. Note that for spot/shock interaction cases these values change during the interaction; the numbers given here are representative values to indicate trends.

\begin{tabular}{lccc}
\hline \hline Case & $u_{\text {front }}$ & $u_{\text {tail }}$ & Half-spreading angle \\
\hline M2S & 0.87 & 0.53 & $5.0^{\circ}$ \\
M2SS & 0.86 & 0.42 & $18.5^{\circ}$ \\
M4S & 0.87 & 0.59 & $4.0^{\circ}$ \\
M4SS & 0.86 & 0.45 & $11.0^{\circ}$ \\
\hline \hline
\end{tabular}

flows. They also observed a reduction in the trailing edge propagation speed of the spot with increasing adverse pressure gradient.

\section{LATERAL SPREADING MECHANISM}

The present simulations have shown a strong dependence of spot growth rate on Mach number and on pressure gradient in the context of a shock interaction. In this section we develop a physical mechanism of spot growth via lateral shear-layer instability into an empirical model that can predict the overall effects of pressure gradient and Mach number on spot lateral growth rate.

To understand the mechanism of spot growth it is useful to consider what would happen to a localized patch of turbulent fluid, extending throughout the boundary layer and surrounded by laminar fluid [Fig. 19(a)]. By convection, structures towards the outer edge of the boundary layer will move faster and form the leading edge of the spot, including a leading-edge overhang, while the near-wall structures will convect slowly and form the rear of the spot. Turbulent structures at the edge of the boundary layer convect at speeds just below the freestream velocity, while typical self-sustaining structures near the wall occur in the buffer layer, where local mean velocities may be $50 \%$ of the freestream. Thus the leading and trailing edges will move apart as the whole turbulent patch moves downstream, with the interior being filled in with new turbulent structures.

The argument can be taken further by considering typical laminar and turbulent boundary-layer profiles, as shown in Fig. 19(b). Due to the higher friction for turbulent flow there is an extended region where the local streamwise velocity in a turbulent region is higher than in the surrounding

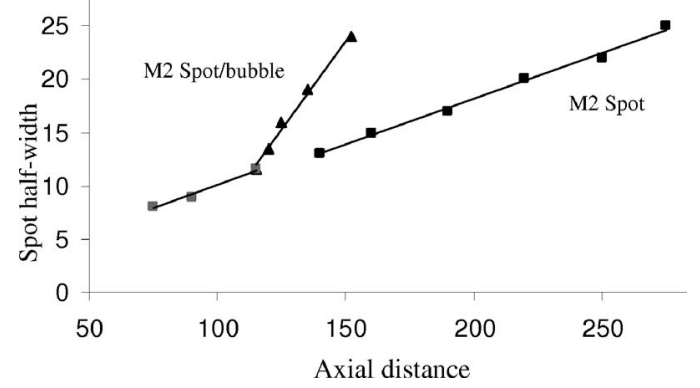

FIG. 17. Lateral spreading of the spot (Mach 2 flow); triangle, spot/bubble interaction; square, isolated spot. 


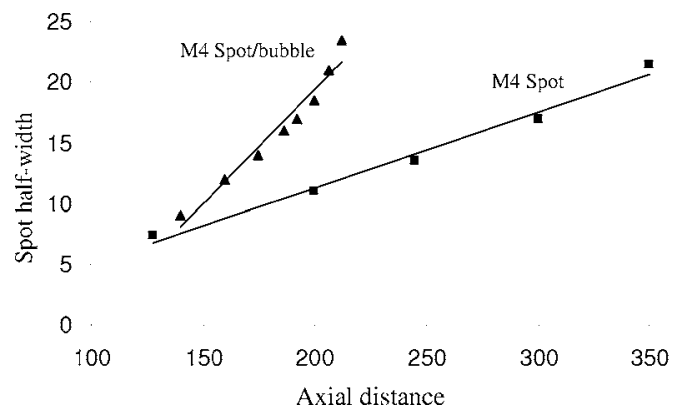

FIG. 18. Lateral spreading of the spot (Mach 4 flow); triangle, spot/bubble interaction; square, isolated spot.

laminar boundary layer. At the rear of the turbulent patch near the wall there must therefore be a strong $\partial u / \partial x$ at the interface between the laminar and turbulent regions. By continuity (neglecting small spanwise variations in $w$ which will scale with overall spot dimensions, rather than the interface) this must be associated with a negative $\partial v / \partial y$ and hence an inrush of fluid, carrying high momentum laminar fluid towards the wall. The resulting laminar profiles are fuller and more stable than the surrounding boundary layer, hence the terminology of "calmed" region. Given that the stabilizing effect is likely to be stronger behind the central spot region than the lateral wingtips (where the turbulent flow is less well established), the rear crescent shape of turbulent spots can be understood. The observed spanwise velocity gradient $\partial w / \partial z$ in spots is positive only near the wingtip region (where spot fluid is ejected in the spanwise direction out of the spot) and so tends to reinforce the effect of $\partial u / \partial x$ in forming the calmed region behind the central spot region.

Next we consider lateral spreading of the spot, as sketched in Fig. 20. The velocity profile $u(z)$ is shown within the near-wall region where the mean turbulent flow velocity is significantly higher than the surrounding laminar flow. We suppose that the lateral spreading of the spot is driven by the inflectional instability of such profiles and that the presence of the mean shear $\partial u / \partial y$ plays an important secondary role. The inflectional instability will tend to develop vertical vorticity and the mean shear will tilt it to give longitudinal vortices, as sketched in Fig. 21. Such vortices are oriented to lift up laminar fluid outside the spot and give local inflection points in $u(y)$ profiles. Such profiles will rapidly break down to turbulence and the process can begin again. This basic mechanism is consistent with the existence of a velocity defect region near the wingtip of the spot (Wygnanski et al. ${ }^{5}$ and Makita and Nishizawa ${ }^{27}$ ). Furthermore, Seifert et ll. $^{25}$ in

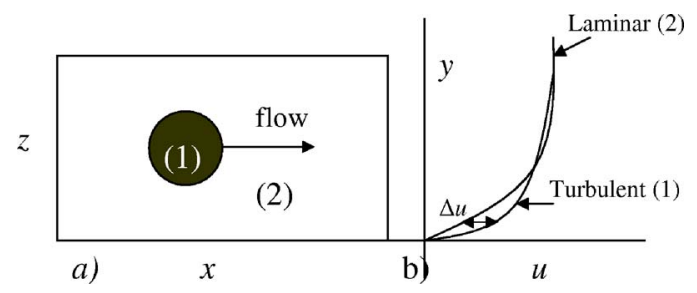

FIG. 19. Schematic of a localized turbulent patch and the associated laminar and turbulent velocity profiles.

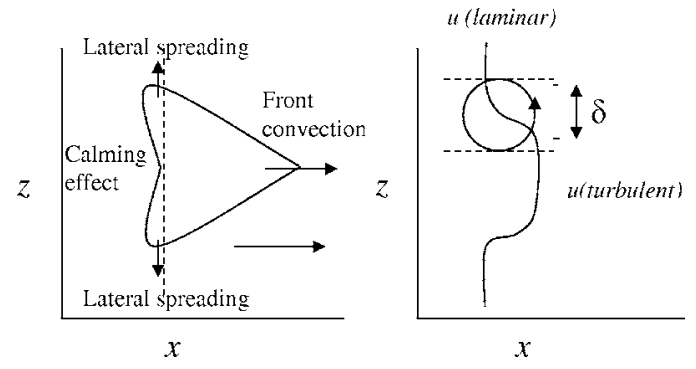

FIG. 20. Schematic illustration of the lateral shear near the wingtips.

their simultaneous measurements of two velocity components in the turbulent spot observed the existence of negative velocity defect near the wingtips which possesses inflectional velocity profiles and Krishnan and Sandham ${ }^{28}$ noticed a stronger upwash near the interacting wingtips during the merging of laterally displaced spots. The latter study also showed the birth of new structures due to the breakdown of the inflectional velocity profiles associated with the velocity defect region.

This mechanism is also consistent with an idea of Liepmann, ${ }^{29}$ who proposed that the lateral contamination could be considered as a mixing process rather than diffusion, and noted a correspondence between lateral turbulence growth rates and the spreading angles of free jets (mixing layers). From the boundary layer profiles shown in Fig. 19(b) we see that at some wall-normal location $y_{m}$ there will be a maximum velocity difference

$$
\Delta u_{m}=\max \left(u_{t}(y)-u_{l}(y)\right)
$$

where $u_{t}(y)$ is the velocity of the turbulent flow inside the spot (assumed to be fully developed) and $u_{l}(y)$ is the velocity of the surrounding laminar flow. We can write the growth rate of spot half-width $b$ as

$$
\frac{d b}{d x}=f(M) \frac{\Delta u_{m}}{u_{t}\left(y_{m}\right)+u_{l}\left(y_{m}\right)},
$$

where $f(M)$ is an empirical function. We do not suppose that the function $f(M)$ is the same as the corresponding function for plane mixing layers, since the presence of a strong $\partial u / \partial y$ will have a modifying effect, so far unstudied by experiment or simulation.

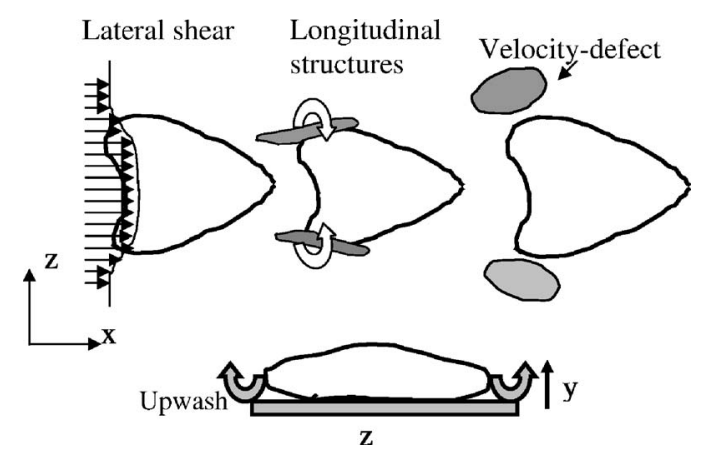

FIG. 21. Schematic of the proposed lateral destabilizing mechanism of the spot. 

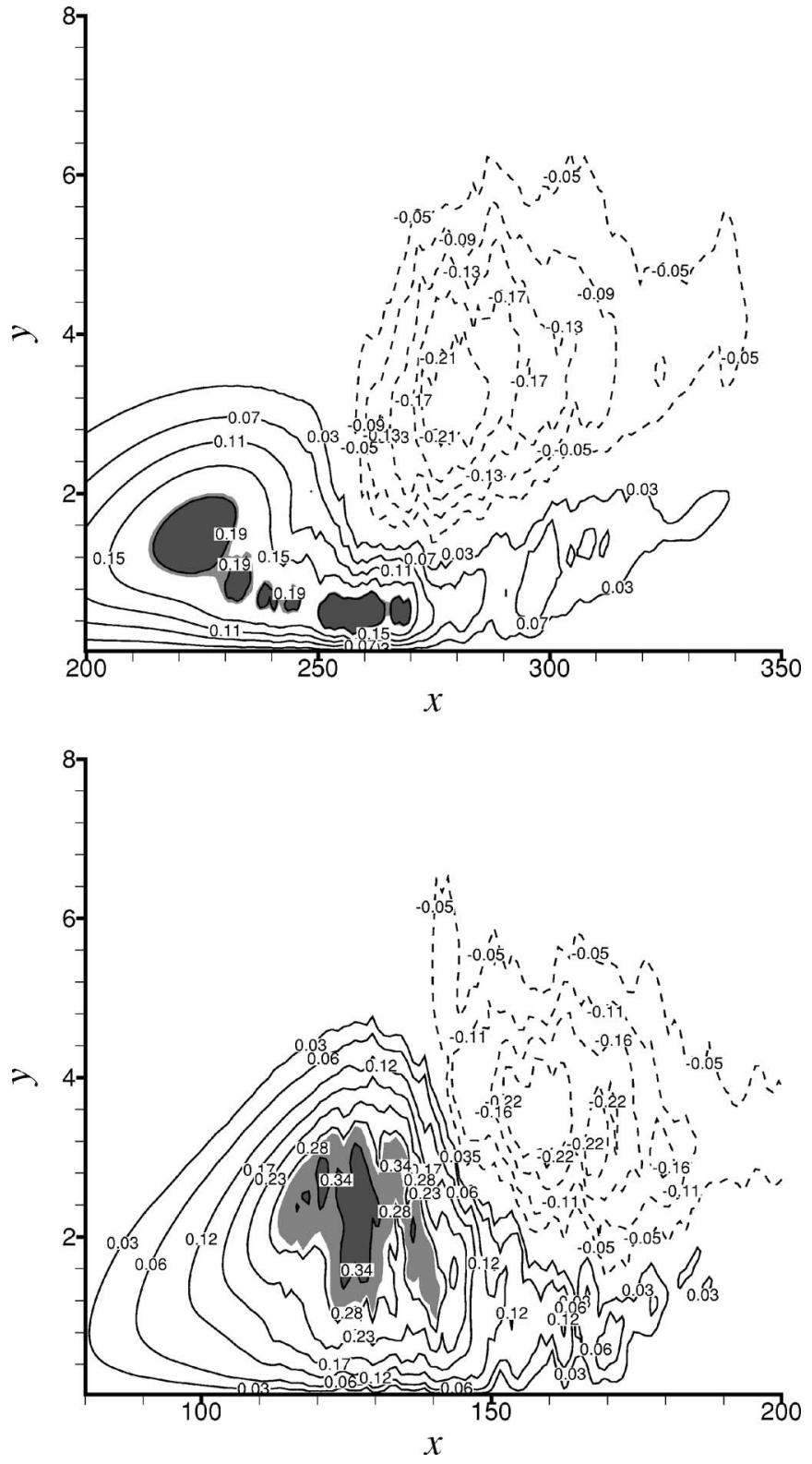

FIG. 22. Velocity difference $u_{t}-u_{l}$ (not to scale). (a) M2S case at $t=391$, (b) M2SS case at $t=234$.

To identify the parameters that control the growth rate, we quantify some of the main features. Figures 22 and 23 show the velocity difference $u_{t}(y)-u_{l}(y)$ between the turbulent flow inside the spot and the surrounding laminar flow for the present spots. The span-averaged velocity field within the turbulent spot was taken as $u_{t}$. The M2SS and the M4SS cases shows a higher velocity difference than the corresponding isolated spot cases (M2S and M4S). The estimated values of the velocity ratio $\Delta u_{m} /\left(u_{t}\left(y_{m}\right)+u_{l}\left(y_{m}\right)\right)$ for the M2S and M2SS cases are 0.28 and 0.72 , respectively. For the M4S and M4SS cases the velocity ratios are 0.32 and 0.67 . This indicates a stronger lateral shear and an increase in the spot spreading rate by a factor of 2.6 (M2SS) and 2.1 (M4SS) due to spot/bubble interactions. These factors are lower than the observed increase in the spot growth rate shown in Table III, suggesting that the spanwise velocity component induced by the structures near the wingtip region also contributes to an
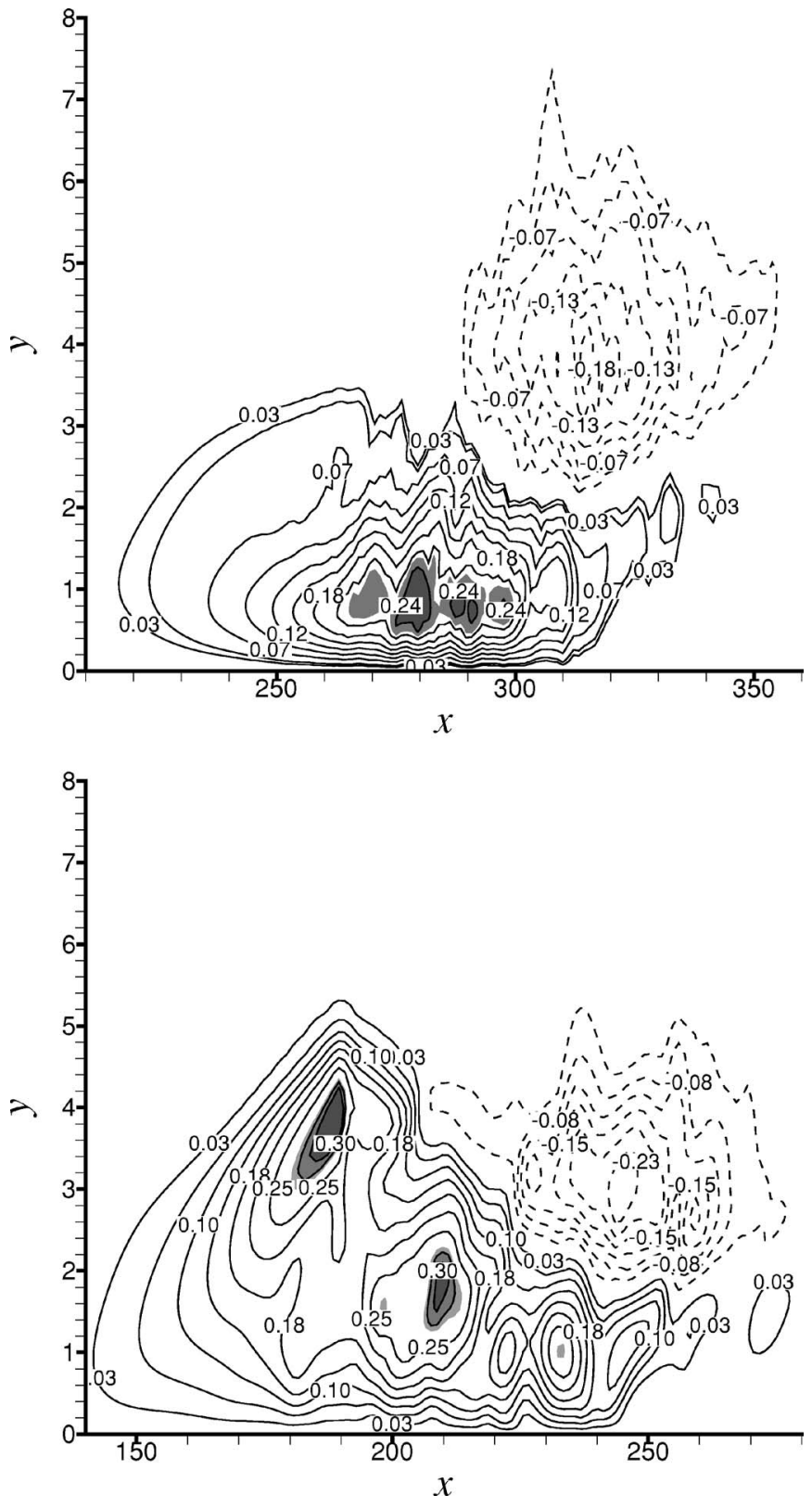

FIG. 23. Velocity difference $u_{t}-u_{l}$ (not to scale). (a) M4S case at $t=390$, (b) M4SS case at $t=300$.

increase in the spot spreading rate (Seifert et al. ${ }^{25}$ ).

The proposed mechanism based on lateral shear qualitatively explains variation in spot spreading rate in a variety of flows as follows:

(i) In flows with pressure gradient the laminar profile is more sensitive than the turbulent profile. With favorable pressure gradient the laminar velocity profile is fuller leading to a reduced $\Delta u_{m}$. Hence the growth rate of the spot is reduced, consistent with Katz et al. ${ }^{10}$

(ii) In an adverse pressure gradient flow, following the same argument, $\Delta u$ increases due to the deceleration of the laminar base flow and an enhanced spot spreading can be obtained, consistent with Seifert and Wygnanski. ${ }^{7}$

(iii) In the experiments of Zilberman et al., ${ }^{30}$ spot growth 
was reduced during spot merging into a full-turbulent boundary layer downstream. In this case the surrounding fluid $u_{l}(y)$ changes to turbulent and $\Delta u_{m}$ reduces, ultimately to zero, leaving normal turbulent diffusion, rather than enhanced lateral growth as the main mechanism.

(iv) In high speed flows, the compressibility effects $[f(M)]$ accounts for the stabilization of the spot breakdown $\left(\right.$ Narasimha $\left.{ }^{4}\right)$. Further detailed spot studies at different Mach numbers will be useful to quantify the compressibility effect, as the function $f(M)$ appears to be quantitatively different to that seen in free shear layers.

\section{SUMMARY}

The present DNS study has demonstrated the complex dynamics of turbulent spot/separation bubble interactions in a compressible wall-bounded flow. The spot core plays an active role in collapsing the bubble, while the spot overhang remains passive. The interaction of a turbulent spot with a shock-induced separation bubble considerably enhances the spot spreading. This is expected to play a major role in accelerating the transition process in high-speed flows with strong compressibility effects. The spot/bubble interaction is also found to amplify streamwise vortices at the rear of the spot as it passes the reattachment region of the bubble. The present study also confirms that the laminar flow separation in adverse pressure gradient flows can be prevented by triggering transitional/turbulent spots in the flow. A destabilizing mechanism associated with the spot spreading in the lateral direction is obtained from the present DNS results. This mechanism, based on the lateral shear layer instability, qualitatively explains the spot dynamics in a variety of flows.

\section{ACKNOWLEDGMENT}

The authors would like to acknowledge the financial support of the European Space Agency (ESA-ESTEC) for this work, under Contract No. 17531/03/NL/SFe, technical officer Dr. J. Steelant.

${ }^{1}$ H. W. Emmons, "The laminar-turbulent transition in a boundary layer," J. Aeronaut. Sci. 18, 490 (1951).

${ }^{2}$ S. Dhawan and R. Narasimha, "Some properties of boundary layer flow during transition from laminar to turbulent motion," J. Fluid Mech. 3, 418 (1958).

${ }^{3}$ G. B. Schubauer and P. S. Klebanoff, "Contribution on the mechanics of boundary-layer transition," NACA Technical Note 3489 (1955).

${ }^{4}$ R. Narasimha, "The laminar-turbulent transition zone in the boundary layer," Prog. Aerosp. Sci. 22, 29 (1985).

${ }^{5}$ I. Wygnanski, M. Sokolov, and D. Friedman, "On a turbulent spot in a laminar boundary layer," J. Fluid Mech. 78, 785 (1976).

${ }^{6}$ E. Gutmark and R. F. Blackwelder, "On the structure of a turbulent spot in a heated laminar boundary layer," Exp. Fluids 5, 217 (1987).

${ }^{7}$ A. Seifert and I. Wygnanski, "On turbulent spots in laminar boundary layer subjected to self-similar adverse pressure gradient," J. Fluid Mech. 296, 185 (1995).

${ }^{8}$ B. Cantwell, D. Coles, and P. Dimotakis, "Structure and entrainment in the plane of symmetry of a turbulent spot," J. Fluid Mech. 87, 641 (1978).

${ }^{9}$ M. Gad-El-Hak, R. F. Blackwelder, and R. J. Riley, "On the growth of turbulent regions in laminar boundary layers," J. Fluid Mech. 110, 73 (1981).

${ }^{10}$ Y. Katz, A. Seifert, and I. Wygnanski, "On the evolution of turbulent spot in a laminar boundary layer in favorable pressure gradient," J. Fluid Mech. 221, 1 (1990)

${ }^{11}$ S. Zhong, C. Kittichaikan, H. P. Hodson, and P. T. Ireland, "Visualization of turbulent spots under the influence of adverse pressure gradients," Exp. Fluids 28, 385 (2000).

${ }^{12}$ M. C. Fischer, "Spreading of a turbulent disturbance," AIAA J. 10, 957 (1972).

${ }^{13}$ J. P. Clark, T. V. Jones, and J. E. LaGraff, "On the propagation of naturally-occurring turbulent spots," J. Eng. Math. 28, 1 (1994).

${ }^{14}$ D. J. Mee, "Boundary-layer transition measurements in hypervelocity flows in a shock tunnel," AIAA J. 40, 1542 (2002).

${ }^{15}$ A. Pagella, U. Rist, and S. Wagner, "Numerical investigation of smallamplitude disturbances in a boundary layer with impinging shock wave at Ma=4.8," Phys. Fluids 14, 2088 (2002).

${ }^{16} \mathrm{M}$. Gerritsen and P. Olsson, "Designing an efficient solution strategy for fluid flows: 1. A stable high order finite difference scheme and sharp shock resolution for the Euler equations," J. Comput. Phys. 129, 245 (1998).

${ }^{17}$ M. H. Carpenter, J. Nordstrom, and D. Gottlieb, "A stable and conservative interface treatment of arbitrary spatial accuracy," J. Comput. Phys. 148, 341 (1999).

${ }^{18}$ F. Ducros, V. Ferrand, F. Nicoud, C. Weber, D. Darracq, C. Gacherieu, and T. Poinsot, "Large-eddy simulation of the shock/turbulence interaction," J. Comput. Phys. 152, 517 (1999).

${ }^{19}$ N. D. Sandham, Q. Li, and H. C. Yee, "Entropy splitting for higher-order numerical simulation of compressible turbulence," J. Comput. Phys. 178, 307 (2002).

${ }^{20}$ L. Krishnan, "Dynamics of turbulent spots in a compressible flow," Ph.D. thesis, University of Southampton, U.K., 2005.

${ }^{21}$ P. R. Spalart, "Direct simulations of a turbulent boundary layer up to $R_{\theta}$ =1410," J. Fluid Mech. 187, 61 (1988).

${ }^{22} \mathrm{~J}$. W. Elder, "An experimental investigation of turbulent spots and breakdown to turbulence," J. Fluid Mech. 9, 235 (1960).

${ }^{23}$ A. E. Perry, T. T. Lim, and E. W. Teh, "A visual study of turbulent spots," J. Fluid Mech. 72, 731 (1981).

${ }^{24}$ H. P. Hodson and R. J. Howell, "Bladerow interactions, transition and high-lift airfoils in low-pressure turbines," Annu. Rev. Fluid Mech. 37, 71 (2005).

${ }^{25}$ A. Seifert, M. Zilberman, and I. Wygnanski, "On the simulataneous measurements of two velocity components in the turbulent spot," J. Eng. Math. 28, 43 (1994).

${ }^{26}$ I. Wygnanski, M. Zilberman, and J. H. Haritonidis, "On the spreading of a turbulent spot in the absence of a pressure gradient," J. Fluid Mech. 123, 69 (1982).

${ }^{27}$ H. Makita and A. Nishizawa, "Characteristics of internal vortical structures in a merged turbulent spot," J. Turbul. 2, 1 (2001).

${ }^{28}$ L. Krishnan and N. D. Sandham, "On the merging of turbulent spots in a supersonic boundary-layer flow," Int. J. Heat Fluid Flow 27, 542 (2006).

${ }^{29} \mathrm{H}$. W. Liepmann, "Recent notes on transition between laminar and turbulent flow by transverse contamination," Appendix A in A. J. Chartres, "Transition between laminar and turbulent flow by transverse contamination," NACA TN-891 (1943).

${ }^{30}$ M. Zilberman, I. Wygnanski, and R. E. Kaplan, "Transitional boundary layer spot in a fully turbulent environment," Phys. Fluids 20, S258 (1977). 